\title{
Using New Concepts in Nanoscience to Develop New Materials
}

\author{
Xiang Zhang1,2* \\ ${ }^{1}$ The founder of Cambridge Victoria College and Principal Consultant of Lucideon, Cambridge Science Park, UK \\ ${ }^{2}$ Professor of Sinano (CAS) and Xiangcheng Medical Materials Technology, Suzhou, China
}

*Corresponding author: Xiang Zhang, The founder of Cambridge Victoria College and Principal Consultant of Lucideon, Cambridge Science Park, UK and Professor of Sinano (CAS) and Xiangcheng Medical Materials Technology, Suzhou, China.
Received Date: February 03, 2020

Published Date: February 20, 2020

\section{Opinion}

The foundation of material science has always been largely rooted in chemistry and the elements of periodic table, many of which have not yet been completely studied and whose full potential has still not been determined. In fact, it is possible to view material science not as "a" science in and of itself, but rather a combination of multiple scientific disciplines, including chemistry, physics and mechanics. The science of materials chemistry is closely associated with chemical structures which vary in scale, with small scale atomic, nanometer and micrometer structures typically having greater importance. When studying the characteristics of a new structure including its associated chemical, physical, mechanical properties etc. understanding these properties at multiple scales from atomic, up to micrometer is very important in determining the material's range of functionalities. Completing this research and examining the outcomes greatly improves the ease and successfulness of new material applications and this remains true whether you are working with inorganic materials, organic materials or hybrids of the two. In my opinion, future developments in material science should aim to cross the border of inorganic and organic science, focusing instead on the study of the properties of atomic-micro scale material structures rather than following well established standards, such as ISOs for materials, all at macroscales.

Where should we be looking to in 2020 and beyond? In order to move materials science into new territory material scientists should be thinking about materials in a new way: Size matters and study of material structures begin at the atomic level [1]. Accurately establishing the relationship between material nanostructures (starting at the atomic level) and their properties should be of utmost importance. While the term "Nanotechnology" has become extremely prevalent in the past few decades and is associated with its own branch of technological development, "Nanoscience" in general has seen far less use. Material structures and properties from the atomic to micro scales are generally still poorly investigated and understood, which has become a bottleneck for not only material science, but also technological development and engineering in general. For the remainder of this piece I will aim to provide examples where understanding of nanostructures has contributed to the development of new materials with novel applications.

In the area of metals and alloy materials, controlled study of lower end nanostructures with the aid of computational modelling and engineered realization of controlled nanostructures could create entirely new classes of metallic materials. For example, highentropy nanostructure alloys consisting of five or more elements have considerable development prospects in the next decade [2]. This class of materials offers a range of possibilities for overcoming problems associated with traditional alloys.

In the area of composites, my personal area of expertise, future developments will likely involve innovations in both the design and development of nanostructured composites. For example, we are currently developing a high load-bearing polymer-based hybrid that contains both inorganic and organic constituents. The new nanocomposites will have the potential to become a new generation of implantable medical devices, usable in humans as permanent hip and knees implants. These new nanohybrids have improved biochemical, physiological and, most importantly, mechanical compatibility in comparison to many materials currently being used for knee and hip implants, such as metal, 
ceramic or combinations of the two along with UHMPE (ultra-high molecular weight polyethylene).

Another area where composites are being utilized is in the design and development of nanostructured thermoplastic based carbon fibre or carbon nanotube composites which forgo epoxy resin based thermosetting. These composites are lightweight, extremely strong, tough and have radiation shielding properties giving them potential applications in the aerospace and defense industries.

Bioresorbable polymer-based ceramic nanohybrids also have a well-controlled nanostructure. The evolution from pure polymerbased medical devices to polymer - based ceramic hybrids can meet unmet market needs for better clinical performance over existing polymer systems. My own research is currently focused on the new theories and industry practices of high-performance medical implants, in particular about biomechanics, bio fracture mechanics and bifunctionality. These are directly relevant to the design and development of implants employing bioresorbable polymer-based ceramic hybrids. The typical biodegradable and bioresorbable polymers used in these are polyesters, including polylactide (PLA), polyglycolide (PGA), poly( $\varepsilon$-caprolactone) (PCL), poly (trimethylene carbonate) (PTMC) and poly(para-dioxanone) (PDO) along with their copolymers. The family of bioresorbable ceramic glass mainly consists of phosphorous glass with a range of degradation capacities to suit to different applications [3].

More research on semiconductors and other electronic materials will be also important [4]. Currently this is being driven by the information and computing technology industry, moving towards increasingly complex monolithic integrated devices, higher-performance microprocessors, and chips that take advantage of three-dimensional (3D) layouts development. New devices will need new material technology to combine memory and logic functions, as well as energy-saving structures. Research into materials that enable more efficient power management will be a major focus. Two-dimensional materials, including graphene which has a well-known atomic level nanostructure, provide an opportunity to explore the properties of surface electronic states. The near-atomic layering of such materials, the weak interactions between layers and the existence of natural "flaws" in the material surface provide opportunities for discovery and potential applications for electronic and optical devices.

The properties of topological materials are determined by the topological properties of their excitation spectra, and they will continue to provide a wide range of fields for exploration, with potential for many applications. In the field of ceramics, energysaving processes will be built around manufacturing, which will enable the production of denser and ultra-high-temperature ceramics. The improvement in characterization and processing capabilities opens new opportunities for glass research, which may lead to their use as solid electrolytes for energy storage and nonlinear optics. Composites will become more and more suitable for more advanced applications, far beyond the traditional structural role.

Other promising areas include perovskite that will continue to attract great interest, mainly because of their potential advantages for single junction solar cells. Hybrid nanocomposites have broad application prospects in optoelectronics and photovoltaic conversion technologies due to their constituent particles with good optical properties and high carrier mobility. Multifunctional materials, such as those that provide both structure and thermal management, enhanced communication or sensing capabilities, are an increasingly important part of such materials. Metamaterials are another important category whose structures provide specific functional responses, and they provide huge opportunities in many different technologies, such as energy-efficient light sources, sensing applications, thermal engineering, and microwave technology.

In this article, I have aimed to demonstrate both the usefulness and necessity of "nanoscience" when it comes to the future of materials science and the development of new technology. New areas of material nanoscience should aim to understand materials from the atomic to lower end nanometer scale and future research should apply traditional knowledge of material chemistry, physics, mechanics to nano-scale structures. This will enable us to better understand, control, and discover applications for new, reliable, economically viable materials, ranging from super alloys to polymer composites, that will not only benefit science and industry but also society as a while.

\section{Notes/Comments: R\&D}

\section{High load-bearing polymer-based ceramic hybrids}

New generation of biomaterials needs fundamental understanding the relationship between nanostructure and properties, polymer-based ceramic hybrids that contain inorganic and organic constituents, and the extent to which each constituent affects mechanical properties, biocompatibility and bioactivity in medical applications. Many natural materials are nanocomposites with good properties needed for living species on the earth. The question is what mechanisms operate on the nano-scale? Answers to this question will help in the future design and development of new biomaterials. Bone, which consists largely of natural collagen and hydroxyapatite, is a good example. Understanding the nanocharacteristics and resulting biological responses of 'smart' composite biomaterials will be the key to develop safer and more effective synthetic products in the future.

\section{Bioresorbable polymer-based ceramic hybrids}

New theories and industry practice for design and development of bioresorbable polymer-based ceramic hybrids will be the future new development. The evolution from pure polymer-based medical devices to polymer-based ceramic hybrids can meet unmet market needs for better clinical performance over existing polymer systems. There are many factors that will affect medical implant performance and, historically, most of them have been well studied, 
such as bioactivities and biocompatibility. The area of my research is mainly on new theories and industry practice of high-performance medical implants of biomechanics, bio fracture mechanics and bifunctionality, directly relevant to design and development of such implants employing bioresorbable polymer-based ceramic hybrids. One family is of biodegradable and bioresorbable polymers are polyesters, including polylactide (PLA), polyglycolide (PGA), poly( $\varepsilon$-caprolactone) (PCL), poly (trimethylene carbonate) (PTMC) and poly(para-dioxanone) (PDO) and their copolymers. The family of bioresorbable ceramic glass is mainly on phosphorous glass with a range of degradation capacity to suit to different applications.

\section{Hydroxyapatite based materials}

Hydroxyapatite (HA) is a well-known biomaterial ever used for medical applications since 1969. To make HA to be adapted by different biological species (like cells); we must change its properties. This can be done by multi-substituting different elements, say $>=2$, into HA (mxHA) crystals. As a result, we have changed the charge potential (positive or negative as well as the strength) and surface characteristics. It is now possible to make a series of new HA-based biomaterials by substituting different elements into HA to meet different application needs. One area of the great importance to science and application is to develop mxHA is to make them have positive effect to tissues with better bioactivity activities and, on the other hand, to make them with good antibacterial functionalities with aid of elements substituted in the HA.

\section{Controlled release technology}

Since the first polymer was studied for the controlled release technology (CRT) of drugs in the 1970s, drug safety and efficacy have been improved over the last 40 years. Due to complexity of drug or other active ingredients and delivery systems, polymer CRT has certain limitation and cannot meet all demanding applications like drugs that are difficult or very easy to be dissolved in the body fluid and those macromolecules like protein/peptide. We are developing new inorganic CRT, in particularly in combination with organic CRT, which will play important role for new drug development and reformulation of exiting drugs. There are not shortages of drugs in the world but smart drug delivery systems that can deliver drug/s in a controlled manner.

\section{Mini - medical devices - biosensors}

Healthcare is seen increasingly expensive. Majority cost is for hospitals. To make people rely on hospitals less for diagnostics, biosensor technology for home use and POC is one way forward with advantage of speed, sensitivity and, above all, simplicity. With success of glucose biosensor, new biosensors research is in demanding for diagnostics of cholesterol, triglyceride, HbA1c (glycosylated hemoglobin), uric acid, lactate, $\mathrm{H}_{2} \mathrm{O}_{2}$, as well as blood gas.

\section{Acknowledgement}

None.

\section{Conflict of Interest}

No conflict of interest.

\section{References}

1. Cambridge Victoria College is the trade name of Cambridge Industry College Ltd, UK.

2. (2019) Frontiers of Materials Research: A Decadal Survey, USA.

3. Xiang Zhang (2016) Science and Principles of Biodegradable and Bioresorbable Medical Polymers. Materials and Properties, Elsevier, USA.

4. (2019) Frontiers of Materials Research: A Decadal Survey, USA. 